\title{
Perspectiva para la composición futura de los medios de fiscalización en México
}

\author{
Perspective for the future composition on the means of taxation \\ control in Mexico
}

\author{
Sergio Lagunas Puls*, Elda Leticia León Vite \\ Universidad del Caribe, México
}

Recibido el 12 de enero de 2018; aceptado el 27 de junio de 2018

Disponible en Internet el: 16 de enero de 2020

\section{Resumen}

El objetivo es establecer la composición futura de actos de fiscalización realizados por el Servicio de Administración Tributaria (SAT) empleando Procesos de Markov. Se presentan algunas aplicaciones precedentes de este tipo de métodos y posteriormente se explica su aplicación para obtener la composición futura de la fiscalización federal. Se verifica el ajuste del método, contrastando los estimados para los meses de enero a junio del 2017 de los cuales se dispuso de los datos reales; para el resto de los meses se obtuvieron las estimaciones conforme al método, conociendo la expectativa para los métodos en que fiscalizaría el SAT durante todo el año 2017. En las conclusiones se deja constancia que la forma y en número de actos realizados sí cambiará, reduciendo los métodos tradicionales como las Visitas Domiciliarias, lo que es consistente de acuerdo al uso de la tecnología como los Comprobantes Fiscales Digitales, Declaraciones Informativas y Timbrado de Nómina, entre otros.

Código JEL: M48, M42, C13

Palabras clave: Fiscalización; Procesos de Markov; Auditoría

\footnotetext{
*Autor para correspondencia.

E-mail address: slagunas@ucaribe.edu.mx (S. Lagunas Puls).

La revisión por pares es responsabilidad de la Universidad Nacional Autónoma de México. 


\title{
S. Lagunas Puls y E. L. León Vite / Contaduría y Administración 65(2) 2020, 1-18 \\ http://dx.doi.org/10.22201/fca.24488410e.2018.1867
}

\begin{abstract}
The objective is to establish the future composition of control acts performed by the Tax Administration Service (SAT) using Markov Processes. Some previous applications of this type of methods are presented and later their application is explained to obtain the future composition of the federal control. The adjustment of the method is checked, comparing the estimates for the months of January to June 2017, of which the actual data were available; for the rest of the months, estimates were obtained according to the method, knowing the expectation for the methods in which the SAT would supervise throughout the year 2017.

The conclusions state that the form and number of acts performed will change, reducing traditional methods such as home visits, which is consistent according to the use of technology such as Digital Tax Receipts, Informative Statement and Payroll Stamps and others.
\end{abstract}

JEL code: $\mathrm{M} 48, \mathrm{M} 42, \mathrm{C} 13$

Keywords: Taxation; Markov chains; Audit

\section{Introducción}

En México, los actos de fiscalización tienen su origen en los principios constitucionales, señalando que toda contribución que se establezca en el país, debe tener obligatoriedad, proporcionalidad y equidad, tal y como lo contempla la Constitución Política de los Estados Unidos Mexicanos, en su Artículo 31 Fracción IV, señalando la obligación de los mexicanos de contribuir al gasto público de la Federación, Estados y Municipios, de manera proporcional y equitativa que dispongan las leyes (Congreso de la Unión, 2017a).

Otro de los ordenamientos jurídicos que originan actos de fiscalización es el Código Fiscal de la Federación (CFF), que junto con las leyes y reglamentos para el Impuesto Sobre la Renta (ISR), Impuesto al Valor Agregado (IVA), entre otros, contienen normas que fungen como instrumentos de administración tributaria, las cuales constituyen un marco jurídico integrador de conceptos tributarios genéricos, siendo receptáculos de derechos y obligaciones de los contribuyentes pero también para la autoridad (Congreso de la Unión, 2017b).

Dentro de las Disposiciones Generales contenidas en el CFF, se hace hincapié que tanto las personas físicas como las personas morales, están obligadas a contribuir para el gasto público conforme a las leyes fiscales respectivas, siendo que, para verificar el correcto cumplimiento del pago de impuestos federales, la Secretaría de Hacienda y Crédito Público (SHCP), a través del Servicio de Administración Tributaria (SAT), tiene facultades para llevar a cabo los distintos análisis que permiten evaluar el cumplimiento en los pagos; a instancia del SAT se 
desenvuelven las relaciones sustantivas que hacen posible el pago de impuestos, los procedimientos administrativos para hacer efectivo el cumplimiento también del pago, así como la adjudicación de responsabilidades y sanciones que derivan del ejercicio de sus facultades.

No obstante al marco legal, los esfuerzos para llevar a cabo fiscalización en México han sido muy diversos, por citar una breve síntesis se puede decir que desde los años 80 y hasta finales de la década de los años 90, los actos de fiscalización llevados a cabo por la autoridad, que podían ser considerados como convencionales y en cierto modo como de costumbre, además de los informes por Dictamen Fiscal, fueron las Visitas Domiciliarias, llevadas por el personal actuante de la autoridad en los domicilios fiscales de los contribuyentes, y también las Revisiones de Gabinete, en las cuales la autoridad solicitaba que el contribuyente o su representante legal acudiera a sus oficinas con el fin de proporcionar documentación o información que se juzgaba necesaria para verificar el correcto cumplimiento de las obligaciones tributarias.

Durante mediados de los años 90 se tuvo el antecedente de la fiscalización automatizada moderna, con lo que fue la recepción de información de operaciones entre clientes y proveedores así como también de los sueldos y salarios que los patrones pagaban a sus empleados, siendo proporcionada esta información, inicialmente, mediante discos magnéticos (Secretaría de Hacienda y Crédito Público, 1998), para después ser utilizada a manera de compulsas en la búsqueda de discrepancias entre las partes, o bien para verificar, en el caso de las informativas de sueldos, la correcta retención y entero del impuesto aplicado sobre las percepciones de trabajadores.

Durante esa misma época de los años 90, otras instancias facultadas para recaudar contribuciones, como el Instituto Mexicano del Seguro Social (IMSS) y el Instituto del Fondo Nacional de la Vivienda para los Trabajadores (INFONAVIT), iniciaban con el proceso de intercambio de información, incluyendo incluso a los gobiernos municipales para conocer el detalle de las licencias de construcción, permitiendo identificar si se estaba o no enterando impuestos sobre los salarios y, además, apoyándose de forma sustantiva en la opinión del Contador Público en Dictamen Fiscal (Instituto Mexicano del Seguro Social, 1992), no obstante, este intercambio de información en su gran mayoría era contenido de forma documental.

No fue sino hasta finales del siglo XX cuando se hizo público que México tenía una de las recaudaciones más bajas de los países de la Organización para la Cooperación y Desarrollo Económico (Hurtado \& Barret, 2014), presionando a las autoridades fiscales para que iniciaran con lo que hasta ahora podría denominarse como la gran implementación de sistemas automatizados, procurando obtener información para fiscalizaciones mucho más ágiles a la que se tenía a finales de los 90, surgiendo un catálogo amplio en declaraciones informativas, desde las que reportaron listado de conceptos que participaban para la determinación de un impuesto (Instituto Mexicano de Contadores Públicos, 2009), como lo fue el ahora derogado 
Impuesto Empresarial a Tasa Única (IETU) pero que en su momento fue considerado con respeto a los principios constitucionales (Comisión de Investigación Fiscal, 2010); y la aún vigente Declaración Informativa de Operaciones con Terceros (DIOT), por mencionar solo estos dos ejemplos, en los cuales los contribuyentes ya no presentaría discos magnéticos sino que en su lugar, enviarían archivos adjuntos, por medios electrónicos, conteniendo la información requerida por la autoridad.

De esta manera los actos de fiscalización para inicios del siglo XXI pasaron a formar una pareja cuasi perfecta con los sistemas automatizados (Servicio de Administración Tributaria, 2016), derivando hasta nuestros días en los registros inmediatos de compra y venta de bienes y servicios consignados en un Comprobante Fiscal Digital (CFDI), emitido en el momento en que ocurre una transacción mercantil e inclusive laboral, con lo que se conoce como timbrado de nómina que no es otra cosa más que otro comprobante digital pero específico al pago por producto de una relación subordinada.

Otro aspecto importante a considerar es que los mecanismos para que la autoridad, específicamente el SAT, se allegase de un mayor volumen de información para evaluar transacciones de los contribuyentes, fueron fortalecidos debido al proceso internacional para el intercambio de información, situación que ya estaba siendo profundizada en países como España, Argentina, Chile (García, Rodríguez, \& León, 2012), y que se hicieron imprescindibles derivado de abusos cometidos en el pasado por la facturación entre empresas relacionadas (León \& Lagunas, 2017). Lo anterior llevó a que las autoridades fiscalizadoras, además de recibir información de las empresas con establecimientos en sus países o domiciliadas en ellos, tuvieran también que presentar la información con partes relacionadas en el extranjero, información que mediante convenios para intercambiar información (Instituto de Estudios Fiscales, 2010), podría ser verificada por la autoridad.

De este modo en México, los actos de fiscalización se han convertido en mecanismos ágiles, automatizados, agrupados por categorías específicas que la autoridad pone a disposición del público en general, siendo vigentes aún Visitas Domiciliarias, Revisiones de Gabinete, Revisiones de Dictámenes, Verificaciones Masivas (que son realizadas de manera automática por los sistemas) y, Revisiones Diversas (focalizadas previo análisis de expedientes de contribuyentes).

Con esta agrupación, la autoridad presenta por meses, los actos de fiscalización desarrollados, los cuales serán considerados para este artículo por el período enero del año 2012 y hasta julio del 2017 (Servicio de Administración Tributaria, 2017) surgiendo la siguiente interrogante a responder al final de este artículo:

¿Debido a los implementos de la tecnología empleados por el SAT, las formas de fiscalizar han cambiado en los últimos meses, o mejor aún, pude esperarse que en corto o mediano plazo cambien? 
Para responder a la pregunta anterior, en los siguientes apartados se presenta un modelo adaptado a los actos de fiscalización, de tipo estocástico, por considerar en estudios precedentes, que este tipo de métodos son adecuados cuando los estimados futuros estarán en función del patrón de comportamiento observado, considerado también como de tiempo continuo (Ordoñez, Lerma, \& Ocampo, 2008).

En el ámbito de la economía y las finanzas han sido sugeridos en algunas ocasiones por encima de los modelos deterministas (Hernández-Lerma \& Venegas-Martínez, 2012), siendo entonces que esta recomendación, por ejemplo, aplique a sustituirlos en lugar de los modelos lineales, con sujeción de que una variable se comporte de una u otra forma condicionada a la influencia de otra y otras variables relacionadas.

\section{Algunas aplicaciones mediante modelos markovianos}

Propuestos originalmente por el científico ruso Andrei Markov, los modelos estocásticos que llevan su apellido han mostrado ser de gran aplicación, reconociéndole el aporte de probabilidades encadenadas (Cadenas de Markov), las cuales hacen referencia a la sucesión de probabilidades en donde un valor futuro dependerá de las probabilidades anteriores, siendo la denominación más común el referirse a las Cadenas de Markov (Bell, 2012; University of St Andrews, 2017; Norris, 1998), esta característica de obtener estimados futuros con base a hechos anteriores, ha sido compartida por otros investigadores, reconociendo todo ellos que los modelos markovianos son apropiados para establecer escenarios futuros en función a las probabilidades acontecidas o presentes (Ching, Huang, Ng, \& Siu, 2013; Hernández-Lerma \& Venegas-Martínez, 2012).

Más allá de una apreciación general, los métodos de Markov han servido para establecer la transición de un estado a otro dentro de posibilidades finitas (Baum, Petrie, Soules, \& Weiss, 2014; Levin, Peres, \& Wilmert, 2007), como por ejemplo, lo aplicado para obtener escenarios futuros en la distribución de los ingresos en una sociedad, logrando obtener interesantes hallazgos tales como afirmar la casi permanente inequidad en la distribución de la riqueza, como lo que acontece en Colombia (Galvis, 2015; Quah, 1996).

Una sugerencia interesante para los modelos markovianos es emplearlos como alternativa de otros modelos más comunes, tales como las regresiones, como lo manifestado por Hernández del Valle (2009) en donde propone y desarrolla un interesante análisis valorando la contracción o crecimiento que podía esperarse en economías como las de México y Estados Unidos, el autor mediante un modelo de Markov responde a la pregunta acerca de cuál sería la expectativa para el crecimiento estacionario en México y en Estados Unidos de América, encontrando que por desgracia, en el caso de México se debe esperar un estancamiento en 
poco crecimiento menor a $2 \%$, mientras que, por otra parte, establece que las contracciones a registrarse en los Estados Unidos no estarían más allá del $3 \%$.

En el ámbito financiero los modelos markovianos han demostrado ser eficientes. Un estudio aplicado a la calificación crediticia permitió integrar un modelo con datos acontecidos de calificaciones crediticias y, a partir de éstos, la forma para establecer las matrices de probabilidad que llevaran, junto con calificaciones o estados actuales, a establecer las calificaciones crediticias futuras; se considera que este estudio es de gran valor ya que describe de una forma matemáticamente clara, el desarrollo y aplicación de los modelos presentados, además de que en su apartado 4 presenta ejemplos concretos aplicados al ámbito financiero (Israel, Rosenthal, \& Wei, 2001).

También es posible apreciar el gran valor que tienen los modelos de Markov cuando se observa que su empleo no solo llega al uso económico o financiero sino a temas coyunturales de la vida, tal es el caso de trabajo aplicado a la demanda del agua potable que será requerida por la población en el corto y mediano plazo; los autores Gagliardi, Alvisi, Kapelan y Franchini (2017) presentan un planteamiento que enfatiza en dos formas, la manera de emplear los modelos markovianos, la primera representada por modelos homogéneos (Darling \& Siegert, 1953), en donde la matriz de probabilidad y sus datos, permanecen constantes (continuos) para obtener escenarios próximos, siendo la única variante los valores de un estado actual determinado, mientras que la segunda manera consiste en variar la matriz de probabilidad conforme se obtengan nuevos valores (Aziz, Sanwal, Shingal, \& Brayton, 2000).

Otros autores (Gagliardi, Alvisi, Kapelan, \& Franchini, 2017; Ching, Huang, Ng, \& Siu, 2013) precisan que un modelo no homogéneo es aquel en donde varia el estado actual pero también los valores de las matrices de probabilidad, de ahí denominada también como matriz de transición en donde los datos se ajustan en diferentes períodos de tiempo, los autores concluyen que ambos tipos de modelos de Markov pueden ser aplicados a casos específicos de la vida real como lo pudieron constatar en su estudio aplicado a la demanda del agua (Gagliardi, Alvisi, Kapelan, \& Franchini, 2017).

Otro ejemplo interesante se aplicó a la previsión de los vientos para corto y mediano plazo, en este planteamiento los autores decidieron emplear matrices de probabilidad no homogéneas, es decir, con variantes en los valores de probabilidad para distintos períodos de tiempo, concluyendo que un modelo markoviano, discreto y con matrices de probabilidad variables permiten predecir de una manera clara y relativamente sencilla los escenarios futuros para las velocidades del viento (Carpinone, Langella, \& Testa, 2015). 


\section{Forma para estimar los actos de fiscalización mediante modelo de Markov}

Se considera la categorización de los actos de fiscalización que realiza el Servicio de Administración Tributaria (SAT) distinguiendo los cinco métodos en que los agrupa: Visitas Domiciliarias, Revisiones de Gabinete, Revisiones de Dictámenes, Verificaciones Masivas y Revisiones Diversas (Servicio de Administración Tributaria, 2017).

Con la información disponible desde enero del año 2012 y hasta diciembre del año 2016, para analizar las expectativas que integren la desagregación de actos de fiscalización, se aplica el método conocido como Matrices de Transición (Render, Stair, \& Hanna, 2012; Anderson, Sweeney, Williams, Camm, \& Martin, 2011), el cual necesita información previa de las variables a analizar que integrarán lo que se conoce como Matrices Estocásticas o de Transición, cuya forma general se representa a continuación

Matriz de transición (1)

$$
P=\left(\begin{array}{ccccc}
P_{11} & P_{12} & P_{13} & \cdots & P_{1 n} \\
P_{21} & P_{22} & P_{23} & \cdots & P_{2 n} \\
\vdots & \vdots & \vdots & \vdots & \vdots \\
P_{m 1} & \cdots & \cdots & \cdots & P_{m n}
\end{array}\right)
$$

así como también el estado actual que guarda la condición de las variables de interés

Vector de probabilidades para un estado actual o presente

$$
\pi(0)=\left(\pi_{1}, \pi_{2}, \pi_{3}, \ldots, \pi_{n}\right)
$$

para que, a partir de estos dos elementos se pueda obtener lo que se conoce como estados fututos o expectativas para la variable 


$$
\pi(i)=\left(\pi_{1}, \pi_{2}, \pi_{3}, \ldots, \pi_{n}\right)
$$

Para la aplicación específica en los métodos de fiscalización, un vector de probabilidades, para expresar la conformación en un momento en el tiempo se representaría como sigue:

Representación de un período futuro $\quad(4)^{l}$

$$
\pi(i)=\pi_{\gamma_{i}}, \pi_{\vartheta_{i}}, \pi_{\varphi_{i}}, \pi_{\omega_{i}}, \pi_{\delta_{i}}
$$

En donde

$$
\begin{aligned}
& \pi(i)=\text { vector de probabilidades para período futuro } \\
& \pi_{\gamma_{i}}= \text { probabilidad para visistas domiciliarias en período futuro } \\
& \pi_{\vartheta_{i}}=\text { probabilidad para revisiones de gabinete en período futuro } \\
& \pi_{\varphi_{i}}=\text { probabilidad para revisión de dictámenes en período futuro } \\
& \pi_{\omega_{i}}=\text { probabilidad para revisiones masivas en período futuro } \\
& \pi_{\tau_{i}}=\text { probabilidad para verificaciones diversas en período futuro }
\end{aligned}
$$

Representación de un período presente $\quad(5)^{2}$

$$
\pi(0)=\pi_{\gamma_{0}}, \pi_{\vartheta_{0}}, \pi_{\varphi_{0}}, \pi_{\omega_{0}}, \pi_{\delta_{0}}
$$

En donde

$$
\begin{aligned}
& \pi(0)=\text { vector de probabilidades para período presente } \\
\pi_{\gamma_{0}}= & \text { probabilidad para visistas domiciliarias en período presente } \\
\pi_{\vartheta_{0}}= & \text { probabilidad para revisiones de gabinete en período presente } \\
\pi_{\varphi_{0}}= & \text { probabilidad para revisión de dictámenes en período presente } \\
\pi_{\omega_{0}}= & \text { probabilidad para revisiones masivas en período presente } \\
\pi_{\tau_{o}}= & \text { probabilidad para verificaciones diversas en período presente }
\end{aligned}
$$

\footnotetext{
${ }^{1}$ Para este trabajo el subíndice i será sustituido por el mes determinado del año 2017 que se estaría estimado.

${ }^{2}$ Para este trabajo el subíndice 0 será sustituido por la indicación mes correspondiente al año 2016 que servirá de período presente.
} 
Para obtener expectativas, se construyen matrices que habrán de cumplir características concretas, la primera es que para todos los momentos y probabilidades, los valores deben adoptar la característica de ser no negativos $p_{\text {mes,metodo de fiscalizacion }}>0$ otra característica es que para cada momento y su conformación de acuerdo a los tipos de actos de fiscalización, organizados por renglones, será igual al 100\% también expresado como $\sum p_{\text {mes,metodos de fiscalizacion }}=1$ cumpliéndose lo anterior para cada uno de los cinco renglones que representan los estados en distintos períodos de tiempo.

La representación de una matriz estocástica o de transición $k x k$ para métodos de fiscalización es la siguiente:

Matriz de transición por método de fiscalización

$$
P=\left(\begin{array}{llllll}
p_{\gamma_{\text {mes 2012 }}} & p_{\vartheta_{\text {mes 2012 }}} & p_{\varphi_{\text {mes 2012 }}} & p_{\omega_{\text {mes 2012 }}} & p_{\tau_{\text {mes 2012 }}} \\
p_{\gamma_{\text {mes 2013 }}} & p_{\vartheta_{\text {mes 2013 }}} & p_{\varphi_{\text {mes 2013 }}} & p_{\omega_{\text {mes 2013 }}} & p_{\tau_{\text {mes 2013 }}} \\
p_{\gamma_{\text {mes 2014 }}} & p_{\vartheta_{\text {mes 2014 }}} & p_{\varphi_{\text {mes 2014 }}} & p_{\omega_{\text {mes 2014 }}} & p_{\tau_{\text {mes 2014 }}} \\
p_{\gamma_{\text {mes 2015 }}} & p_{\vartheta_{\text {mes 2015 }}} & p_{\varphi_{\text {mes 2015 }}} & p_{\omega_{\text {mes 2015 }}} & p_{\tau_{\text {mes 2015 }}} \\
p_{\gamma_{\text {mes 2016 }}} & p_{\vartheta_{\text {mes 2016 }}} & p_{\varphi_{\text {mayo 2016 }}} & p_{\omega_{\text {mes 2016 }}} & p_{\tau_{\text {mes 2016 }}}
\end{array}\right)
$$

Los componentes de la matriz de transición anterior en cada uno de los renglones, representan la conformación porcentual (probabilidad inicial) del número de fiscalizaciones por cada método con respecto al total de fiscalizaciones del mes y año que se indica, utilizando esta información como uno de los elementos para estimar lo que podría esperarse para todos los meses del año $2017^{3}$ (estados futuros). Se conformarán matrices para cada mes que se desee estimar, por ejemplo, para obtener la expectativa de enero 2017 (estado futuro), la matriz se integrará con la conformación de actos de fiscalización de los meses de enero del año 2012 y hasta enero del año 2016.

Los períodos presentes iniciarán con la conformación del mes que corresponda pero del año $2016 \pi(0)_{\text {enero } 2016}$, por lo que el resultado de la expectativa para ese mes pero del 2017 estará denotado por $\pi(i)_{\text {enero } 2017}=\pi(0)_{\text {enero } 2016} P$ de esta manera, subsecuentemente hasta encontrar la expectativa para el mes de diciembre $\pi(i)_{\text {diciembre } 2017}=\pi(0)_{\text {diciembre } 2016} P$

Por lo anterior, se podrán obtener expectativas, identificando si los nuevos mecanismos automatizados del SAT desde la emisión de Comprobantes Fiscales Digitales (CFDI), pasando por timbrado de nómina y hasta información de operaciones con terceros (DIOT), entre otros, hubieren contribuido con algún cambio en la composición del total de actos de fiscalización (Servicio de Administración Tributaria, 2017).

\footnotetext{
${ }^{3}$ Los resultados obtenidos de enero a junio servirán para valorar el error del modelo propuesto ya que a la fecha de este documento se cuenta con la conformación de fiscalizaciones por método al mes de junio 2017.
} 
Notación para obtención de expectativas mensuales futuras (7)

$\pi(i)_{m e s ~ 2017}=\pi(0)_{m e s ~ 2016} P$

$\pi(i)_{\text {mes } 2017}$

$=\left[\pi_{\gamma}(0)_{\text {mes 2016 }}, \pi_{\vartheta}(0)_{\text {mes 2016 }}, \pi_{\varphi}(0)_{\text {mes 2016 }}, \pi_{\omega}(0)_{\text {mes 2016 }}, \pi_{\delta}(0)_{\text {mes 2016 }}\right] P$

\section{Estimados futuros por método de fiscalización}

A continuación, se agrega la Tabla 1 que expresa los datos oficiales para establecer los valores de la matriz de transición correspondiente al mes de enero ${ }^{4}$,

Tabla 1

Número de fiscalizaciones conforme a método

Meses de Enero

Método

$\begin{array}{ccccc}\text { Visitas } & \text { Revisiones de } & \text { Revisiones de } & \text { Revisiones } & \text { Verificaciones } \\ \text { domiciliarias } & \text { gabinete } & \text { Dictámenes } & \text { masivas } & \text { diversas }\end{array}$

\begin{tabular}{lrrrrr} 
Año & \multicolumn{7}{c}{} & \\
\hline 2012 & 1,569 & 787 & 267 & 1,885 & 1,156 \\
2013 & 1,335 & 601 & 295 & 3,127 & 1,183 \\
2014 & 1,240 & 615 & 187 & 1,681 & 237 \\
2015 & 1,245 & 532 & 114 & 2,884 & 528 \\
2016 & 1,059 & 398 & 81 & 707 & 2,089 \\
\hline
\end{tabular}

Fuente: elaboración propia con datos del SAT (2017)

Por lo tanto, al estructurar los valores conforme a la notación (3), la matriz de transición y su gráfica se establecen como sigue:

$$
P_{\text {enero }}=\left(\begin{array}{lllll}
0.2770 & 0.1389 & 0.0471 & 0.3328 & 0.2041 \\
0.2041 & 0.0919 & 0.0451 & 0.4781 & 0.1809 \\
0.3131 & 0.1553 & 0.0472 & 0.4245 & 0.0598 \\
0.2348 & 0.1003 & 0.0215 & 0.5438 & 0.0996 \\
0.2443 & 0.0918 & 0.0187 & 0.1631 & 0.4820
\end{array}\right)
$$

${ }^{4}$ Los valores que corresponden al renglón del año 2016 representarán el estado inicial

$\pi(0)_{\text {enero }} 2016$ que junto con la matriz servirá para estimar el estado futuro de ese mismo mes, pero del año 2017

$\pi(i)_{\text {enero }} 2017$ 


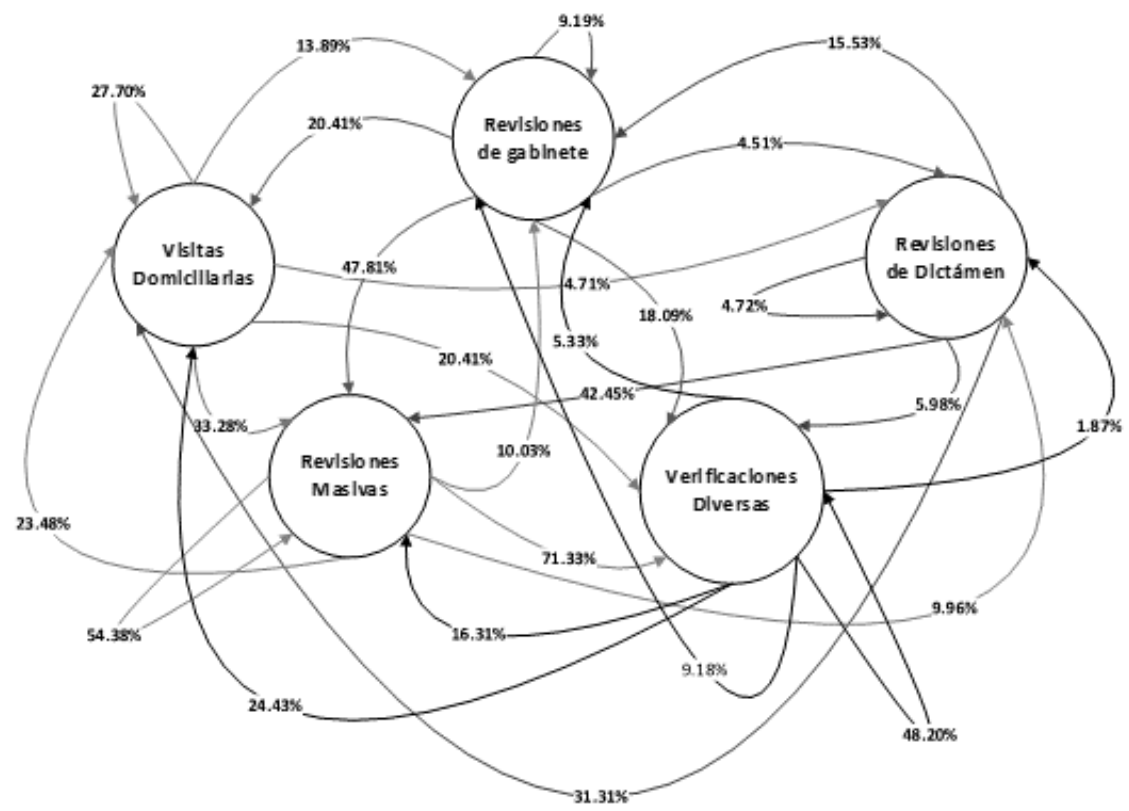

Figura 1. Enero. Representación de probabilidades de transición (enero 2012, enero 2013...enero 2016) de acuerdo a matriz, para estimar Enero 2017

Fuente: Elaboración propia

Ahora bien, como se indicó en la nota de página anterior, la conformación desagregada de $\pi(0)_{\text {enero } 2016}$ es la siguiente:

$$
\begin{aligned}
& \pi_{\gamma}(0)_{\text {enero } 2016}=0.2443 \pi_{\vartheta}(0)_{\text {enero 2016 }}=0.0918 \pi_{\varphi}(0)_{\text {enero } 2016}=0.0187 \\
& \pi_{\omega}(0)_{\text {enero 2016 }}=0.1631 \pi_{\delta}(0)_{\text {enero 2016 }}=0.4820
\end{aligned}
$$


Por lo tanto, utilizando la notación para obtención de expectativas mensuales futuras (3), se obtiene:

$$
\begin{aligned}
& \pi(i)_{\text {enero } 2017} \\
& =\left[\pi_{\gamma}(0)_{\text {enero 2016 }}, \pi_{\vartheta}(0)_{\text {enero } 2016}, \pi_{\varphi}(0)_{\text {enero 2016 }}, \pi_{\omega}(0)_{\text {enero 2016 }}, \pi_{\delta}(0)_{\text {enero 2016 }}\right] P \\
& \pi(i)_{\text {enero } 2017} \\
& =\left[\begin{array}{lllllllll}
0.2443 & 0.0918 & 0.0187 & 0.1631 & 0.4820
\end{array}\right]\left(\begin{array}{lllll}
0.2770 & 0.1389 & 0.0471 & 0.3328 & 0.2041 \\
0.2041 & 0.0919 & 0.0451 & 0.4781 & 0.1809 \\
0.3131 & 0.1553 & 0.0472 & 0.4245 & 0.0598 \\
0.2348 & 0.1003 & 0.0215 & 0.5438 & 0.0996 \\
0.2443 & 0.0918 & 0.0187 & 0.1631 & 0.4820
\end{array}\right) \\
& \pi_{\gamma}(i)_{\text {enero 2017 }}=0.2484 \pi_{\vartheta}(i)_{\text {enero } 2017}=0.1059 \pi_{\varphi}(i)_{\text {enero } 2017}=0.0291 \\
& \pi_{\omega}(i)_{\text {enero 2017) }}=0.3005 \pi_{\delta}(i)_{\text {enero } 2017}=0.3162
\end{aligned}
$$

De acuerdo a lo anterior, se debe esperar que, en el mes de enero 2017, del total de actos de fiscalización, se realice $24.84 \%$ de Visitas Domiciliarias, $10.59 \%$ por Revisiones de Gabinete, 2.91\% de Revisiones de Dictamen, 30.05\% Verificaciones Masivas y 31.62\% Revisiones Diversas.

Cabe aclarar que, para cualquiera de los períodos, es posible representar gráficamente las matrices de transición, sin embargo, por razones de espacio se presentará a continuación, únicamente la imagen que corresponde a una comparativa entre los valores que conformaron la matriz correspondiente a los meses de enero y los valores que correspondieron a los de diciembre (cifras en rojo). 


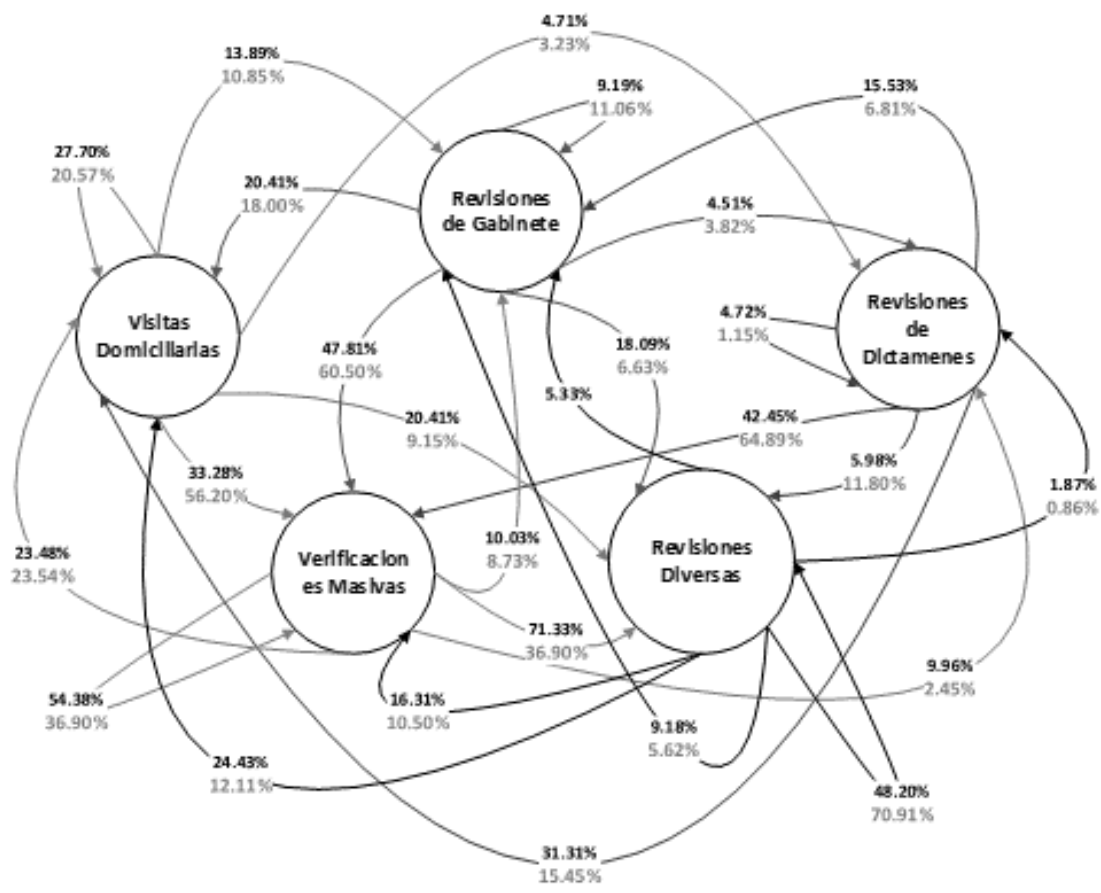

Figura 2. Representación de probabilidades de transición de acuerdo a matrices enero $(2012,2013, \ldots, 2016)$ (negro) - diciembre $(2012,2013, \ldots, 2016)$ (rojo)

Fuente: Elaboración propia

Al comparar las transiciones empleadas para obtener enero 2017 con respecto a diciembre de ese mismo año, se distingue que en dos de los métodos de fiscalización acostumbrados, las Visitas Domiciliarias y las Revisiones de Dictamen, en efecto, sí debe esperarse, para el siguiente apartado del trabajo, una reducción; resultó que para el caso de las Visitas, se espera se reduzca del $27.70 \%$ del total de fiscalizaciones en enero 2017 a solamente $20.57 \%$ para el mes de diciembre, para el caso de la Revisión de Dictamen, la reducción será del 4.72\% del total de actos a solamente $1.15 \%$ lo cual puede interpretarse como una oportunidad para reducir el gasto y aprovechar los medios tecnológicos. 
No obstante, las Verificaciones Masivas, de las cuales podría considerarse como lógico que al menos se mantuvieran en número, o inclusive incrementarse por la tecnología, los resultados indican que se verán reducidas ya que del $54.38 \%$ que representaron en enero 2017, este porcentaje se esperaría alcanzar solamente $36.90 \%$ al cierre del mes de diciembre de ese mismo año. Los métodos que se espera incrementen para el cierre del año 2017, son la Revisiones Diversas ya que de representar en el mes de enero $48.20 \%$ del total de actos de fiscalización, este porcentaje llegaría a 70.91\% para el mes de diciembre, otro de los métodos que se espera sea incrementado lo constituyen las Revisiones de Gabinete pasando del $9.19 \%$ hasta el $11.06 \%$ del total de fiscalizaciones. A partir de las matrices de transición para todos los meses del año, fue posible obtener los estimados completos para los meses de 2017, presentando los resultados en el siguiente apartado.

\section{Resultados y conclusiones}

Inicialmente se presentan aquellos estimados correspondientes a los meses de enero a junio del 2017, los cuales se adoptaron como prueba al confrontarlos con los valores reales, los resultados se presentan en la Tabla 2.

Tabla 2

Confrontación de participación real v.s. estimados por Modelo de Markov

Enero - Junio, 2017

\begin{tabular}{|c|c|c|c|c|c|c|c|c|}
\hline $\begin{array}{c}\text { Método de } \\
\text { Fiscalización }\end{array}$ & Notación & & Enero & Febrero & Marzo & Abril & Mayo & Junio \\
\hline \multirow{3}{*}{$\begin{array}{c}\text { Visitas } \\
\text { Domiciliarias }\end{array}$} & \multirow{3}{*}{$\pi_{\gamma}$} & Real & $24.43 \%$ & $20.72 \%$ & $21.01 \%$ & $18.57 \%$ & $19.73 \%$ & $16.31 \%$ \\
\hline & & Estimado & $24.84 \%$ & $22.12 \%$ & $22.38 \%$ & $20.24 \%$ & $21.00 \%$ & $18.78 \%$ \\
\hline & & $\begin{array}{l}\text { Diferencia } \\
\text { absoluta }\end{array}$ & $0.41 \%$ & $1.40 \%$ & $1.37 \%$ & $1.67 \%$ & $1.27 \%$ & $2.47 \%$ \\
\hline \multirow{3}{*}{$\begin{array}{l}\text { Revisiones de } \\
\text { Gabinete }\end{array}$} & \multirow{3}{*}{$\pi_{\theta}$} & Real & $9.18 \%$ & $7.15 \%$ & $7.18 \%$ & $7.23 \%$ & $7.83 \%$ & $6.42 \%$ \\
\hline & & Estimado & $10.59 \%$ & $8.73 \%$ & $9.00 \%$ & $8.48 \%$ & $8.58 \%$ & $7.68 \%$ \\
\hline & & $\begin{array}{l}\text { Diferencia } \\
\text { absoluta }\end{array}$ & $1.41 \%$ & $1.58 \%$ & $2.18 \%$ & $1.25 \%$ & $0.75 \%$ & $1.26 \%$ \\
\hline \multirow{3}{*}{$\begin{array}{l}\text { Revisiones de } \\
\text { Dictamen }\end{array}$} & \multirow[b]{3}{*}{$\pi_{\varphi}$} & Real & $1.87 \%$ & $1.76 \%$ & $1.94 \%$ & $1.52 \%$ & $1.33 \%$ & $1.38 \%$ \\
\hline & & Estimado & $2.91 \%$ & $2.61 \%$ & $2.66 \%$ & $2.33 \%$ & $2.14 \%$ & $2.06 \%$ \\
\hline & & $\begin{array}{l}\text { Diferencia } \\
\text { absoluta }\end{array}$ & $1.04 \%$ & $0.85 \%$ & $0.72 \%$ & $0.81 \%$ & $0.81 \%$ & $0.68 \%$ \\
\hline \multirow{2}{*}{$\begin{array}{l}\text { Verificaciones } \\
\text { Masivas }\end{array}$} & \multirow{2}{*}{$\pi_{\omega}$} & $\begin{array}{l}\text { Real } \\
\text { Estimado }\end{array}$ & $\begin{array}{l}16.31 \% \\
30.05 \%\end{array}$ & $\begin{array}{l}15.77 \% \\
28.87 \%\end{array}$ & $\begin{array}{l}18.14 \% \\
28.77 \%\end{array}$ & $\begin{array}{l}15.50 \% \\
27.30 \%\end{array}$ & $\begin{array}{l}16.55 \% \\
29.27 \%\end{array}$ & $\begin{array}{l}14.51 \% \\
24.86 \%\end{array}$ \\
\hline & & $\begin{array}{l}\text { Diferencia } \\
\text { absoluta }\end{array}$ & $13.74 \%$ & $13.10 \%$ & $10.63 \%$ & $11.80 \%$ & $12.72 \%$ & $10.35 \%$ \\
\hline \multirow{3}{*}{$\begin{array}{l}\text { Revisiones } \\
\text { Diversas }\end{array}$} & \multirow{3}{*}{$\pi_{\delta}$} & Real & $48.20 \%$ & $54.60 \%$ & $51.74 \%$ & $57.18 \%$ & $54.57 \%$ & $61.39 \%$ \\
\hline & & Estimado & $31.62 \%$ & $37.49 \%$ & $37.20 \%$ & $41.65 \%$ & $39.01 \%$ & $46.61 \%$ \\
\hline & & $\begin{array}{l}\text { Diferencia } \\
\text { absoluta }\end{array}$ & $16.58 \%$ & $17.11 \%$ & $14.54 \%$ & $15.53 \%$ & $15.56 \%$ & $14.78 \%$ \\
\hline
\end{tabular}


Los resultados para los meses que se consideraron como de prueba del modelo, en todos los casos, demostraron congruencia ya que si bien, se esperaba que arrojaran valores distintos por el uso de la tecnología (CFDI, DIOT, timbrado de nómina, entre otros), también no se podían esperar cambios tan abruptos como, por ejemplo, que un método de fiscalización variara en 60 o 70 puntos porcentuales.

Los concentrados de la conformación de los actos de fiscalización para todos los meses del año 2017 se muestran en la Tabla 3.

Tabla 3

Estimados del número de fiscalizaciones conforme a método

Enero - Diciembre 2017

\begin{tabular}{|l|c|r|r|r|r|}
\hline $\begin{array}{c}\text { Meses del año } \\
2017\end{array}$ & $\begin{array}{c}\text { Visitas } \\
\text { Domiciliarias }\end{array}$ & $\begin{array}{c}\text { Revisiones de } \\
\text { Gabinete }\end{array}$ & $\begin{array}{c}\text { Revisiones de } \\
\text { Dictamen }\end{array}$ & $\begin{array}{c}\text { Verificaciones } \\
\text { Masivas }\end{array}$ & $\begin{array}{c}\text { Revisiones } \\
\text { Diversas }\end{array}$ \\
\cline { 2 - 6 } & $\pi_{\gamma}$ & \multicolumn{1}{c}{$\pi_{\vartheta}$} & $\pi_{\varphi}$ & $\pi_{\omega}$ & $\pi_{\delta}$ \\
\hline Enero & $24.84 \%$ & $10.59 \%$ & $2.91 \%$ & $30.05 \%$ & $31.62 \%$ \\
Febrero & $22.12 \%$ & $8.73 \%$ & $2.61 \%$ & $28.87 \%$ & $37.49 \%$ \\
Marzo & $22.38 \%$ & $9.00 \%$ & $2.66 \%$ & $28.77 \%$ & $37.20 \%$ \\
Abril & $20.24 \%$ & $8.48 \%$ & $2.33 \%$ & $27.30 \%$ & $41.65 \%$ \\
Mayo & $21.00 \%$ & $8.58 \%$ & $2.14 \%$ & $29.27 \%$ & $39.01 \%$ \\
Junio & $18.78 \%$ & $7.68 \%$ & $2.06 \%$ & $24.86 \%$ & $46.61 \%$ \\
Julio & $16.43 \%$ & $6.69 \%$ & $1.73 \%$ & $22.94 \%$ & $52.20 \%$ \\
Agosto & $15.91 \%$ & $6.49 \%$ & $1.54 \%$ & $24.04 \%$ & $52.01 \%$ \\
Septiembre & $14.86 \%$ & $6.55 \%$ & $1.64 \%$ & $21.88 \%$ & $55.07 \%$ \\
Octubre & $15.08 \%$ & $6.21 \%$ & $1.57 \%$ & $21.45 \%$ & $55.70 \%$ \\
Noviembre & $15.75 \%$ & $7.19 \%$ & $1.59 \%$ & $21.00 \%$ & $54.47 \%$ \\
Diciembre & $14.70 \%$ & $6.89 \%$ & $1.49 \%$ & $21.19 \%$ & $55.73 \%$ \\
\hline
\end{tabular}

Fuente: Elaboración propia

El modelo adoptado para estimar escenarios arrojó resultados apegados a márgenes congruentes, en todos los casos se obtuvieron cifras futuras sin grandes distorsiones, conservando la tendencia mostrada en los últimos cinco años hacia el apego a la fiscalización más automatizada, derivada del uso de la tecnología más que con los métodos tradicionales, como las visitas domiciliarias.

Es importante destacar que, si los métodos de fiscalización como se sugiere en este trabajo, estarán encaminados a la automatización, entonces debería presentarse un ajuste al gasto destinado a la revisión de contribuyentes, no solo incluyendo las erogaciones salariales, de planta u honorarios, de auditores, supervisores o coordinadores, sino que también se debería esperar una reducción en viáticos. Al término de este trabajo se pudo identificar que, con de acuerdo con el documento "Evolución de la actividad recaudatoria 2016 y Programas y presupuesto 2017", los recursos presupuestales al SAT para el ejercicio 2017 sufrieron una 
reducción del $8 \%$ justificándose esta variación por la optimización lograda por implementos tecnológicos relacionados al pago de impuestos.

Los resultados en este trabajo se contrastaron con los Informes Tributarios y de Gestión que publica trimestralmente el SAT, especialmente el documento al segundo trimestre del 2017, encontrando hallazgos significativos ya que, al comparar año con año, desde el 2012 y hasta el 2017, se identificaron, en las primeras comparativas, incrementos, del año 2012 al 2013 se alcanzó $19.18 \%$ y del año 2013 al 2014 el incremento registrado fue por 34.06\%. No obstante, la comparativa del 2014 respecto al año 2015 resultó en mucho menor recaudación, equivalente a una caída cercana a 45 puntos porcentuales; al continuar la contrastación de resultados se identificó que tampoco lo recaudado en 2016 fue suficiente, al menos, para alcanzar el importe reportado en 2014. De acuerdo a lo anterior, se puede establecer como hipótesis de continuidad para otras investigaciones, que no necesariamente el uso de la tecnología garantiza una mejor eficiencia en la generación de recursos públicos.

Es importante señalar que, si bien la recaudación por actos de fiscalización mostró ser menor a partir del año 2015, por otra parte, la recaudación en general se incrementó, por ejemplo, si se comparan los 916,538.8 millones de pesos correspondientes al año 2014 respecto a los 1,222,128.5 millones de pesos recaudados en el año 2015, entonces se calcula un incremento general del $33.34 \%$ lo cual, para estas conclusiones es interpretado como que el uso de la tecnología propicia que se paguen mejor los impuestos por parte voluntaria más que sean originados por actos de fiscalización.

\section{Referencias}

Anderson, D., Sweeney, D., Williams, T., Camm, J., \& Martin, K. (2011). Procesos de Markov. En D. R. Anderson, Métodos cuantitativos para los negocios (págs. 757-775). México: Cengage Learning.

Aziz, A., Sanwal, K., Shingal, V., \& Brayton, R. (2000). Model Checking Continuous Time Markov Chains. ACM Transactions on Computational Logic (TOCL) Volume 1 Issue 1, 162-170. Disponible en: https://pdfs.semanticscholar.org/d11f/4d4e4318f8cd689df9a0a0e743b69ec7d585.pdf Consultado: 09/10/2017

Baum, L., Petrie, T., Soules, G., \& Weiss, N. (2014). A Maximization Technique Occurring in the Statistical Analysis of Probabilistic Functions of Markov Chains. The Annals of Mathematical Statistics. Vol. 41, No. 1, 164171. Disponible en: http://www.jstor.org/stable/2239727 Consultado: 09/10/2017

Bell, E. (2012). Historia de las metemáticas. México: FCE.

Carpinone, A., Langella, R., \& Testa, A. (2015). Markov chain modeling for very-short-term wind power forecasting. Electric Power Systems Research Vol. 122, 152-158. doi:https://doi.org/10.1016/j.epsr.2014.12.025 Consultado: 03/09/2017

Ching, W., Huang, X., Ng, M., \& Siu, T. (2013). Markov Chains. Models, Algorithms and Applications. USA: Springer. doi:10.1007/978-1-4614-6312-2 


\section{S. Lagunas Puls y E. L. León Vite / Contaduría y Administración 65(2) 2020, 1-18 \\ http://dx.doi.org/10.22201/fca.24488410e.2018.1867}

Comisión de Investigación Fiscal. (2010). Análisis del pronunciamiento emitido por la SCJN con relación a la constitucionalidad del IETU. México: Colegio de Contadores Públicos de México. Disponible en: https://www. ccpm.org.mx/avisos/boletines/Boletin_mayo_2010.pdf Consultado: 14/11/2017

Congreso de la Unión. (10 de 11 de 2017a). Leyes Federales Vigentes. Disponible en: Constitución Política de los Estados Unidos Mexicanos: http://www.diputados.gob.mx/LeyesBiblio/pdf/1_150917.pdf

Congreso de la Unión. (10 de 11 de 2017b). Leyes Federales Vigentes. Disponible en Código Fiscal de la Federación: http://www.diputados.gob.mx/LeyesBiblio/pdf/8_160517.pdf

Congreso de la Unión. (10 de 11 de 2017c). Leyes Federales Vigentes. Disponible en Ley del Impuesto sobre la Renta: http://www.diputados.gob.mx/LeyesBiblio/pdf/LISR_301116.pdf

Congreso de la Unión. (10 de 11 de 2017d). Leyes Federales Vigentes. Disponible en Ley del Instituto de Seguridad y Servicios Sociales de los Trabajadores del Estado: http://www.diputados.gob.mx/LeyesBiblio/pdf/LISSSTE_240316.pdf

Congreso de la Unión. (10 de 11 de 2017e). Leyes Federales Vigentes. Disponible en Ley del Seguro Social: http:// www.diputados.gob.mx/LeyesBiblio/pdf/92_121115.pdf

Congreso de la Unión. (14 de 11 de 2017f). Leyes Federales Vigentes. Disponible en Ley Federal de Procedimiento Contencioso Administrativo: http://www.diputados.gob.mx/LeyesBiblio/pdf/LFPCA_270117.pdf

Darling, D., \& Siegert, A. (1953). The first passage problem for a continuous Markov process. The Annals of Mathematical Statistics, 624-639. The Annals of Mathematical Statistics, 624-639. Disponible en: https://projecteuclid.org/download/pdf_1/euclid.aoms/1177728918 Consultado: 09/10/2017

Gagliardi, F., Alvisi, S., Kapelan, Z., \& Franchini, M. (2017). A Probabilistic Short-Term Water Demand Forecasting Model Based on the Markov Chain. Water, 2-15. Disponible en http://www.mdpi.com/2073-4441/9/7/507 Consultado: 22/09/2017

Galvis, L. (2015). Crecimiento económico y demográfico regional en Colombia, 1985-2012. Revista de Economía Institucional 17(33), 183-201. Disponible en http://www.economiainstitucional.com/esp/vinculos/pdf/No33/ lgalvis.pdf Consultado: 29/10/2017

García, M. C., Rodríguez, A. d., \& León, G. (2012). La fiscalización por parte de las administraciones tributarias de España, Argentina, Chile y México. México: UNAM-ANFECA. Disponible en http://congreso.investiga.fca. unam.mx/docs/xvii/docs/G11.pdf Consultado: 02/08/2017

Hernández del Valle, A. (2009). Método de la cadena de Markov-Remuestreo-Punto de rompimiento estructural del crecimiento económico. El Trimestre Económico vol. LXXVI (3), núm. 303, 619-643. Disponible en http:// www.eltrimestreeconomico.com.mx/index.php/te/article/view/491/529 Consultado: 16/10/2017

Hernández-Lerma, O., \& Venegas-Martínez, F. (2012). Toma de Decisiones de Agentes Racionales con Procesos Markovianos. Avances recientes en economía y finanzas. El Trimestre Económico, LXXIX (4) (316), 733-779. Disponible en http://www.redalyc.org/articulo.oa?id=31340973001 Consultado: 16/10/2017

Hurtado, M., \& Barret, F. (2014). Fiscalización e Impuestos en México. México: PricewaterhouseCoopers. Disponible en https://www.pwc.com/mx/es/impuestos/archivo/2015-02-fiscalizacion.pdf Consultado: 18/10/2017

Instituto de Estudios Fiscales. (2010). Directrices de la OCDE aplicables en materia de Precios de Transferencia a empresas multinacionales y administraciones tributarias. Madrid: OCDE. Disponible en https://read.oecd-ilibrary.org/taxation/directrices-de-la-ocde-aplicables-en-materia-de-precios-de-transferencia-a-empresas-multinacionales-y-administraciones-tributarias-2010_9789264202191-es\#page5 Consultado: 18/10/2017

Instituto Mexicano de Contadores Públicos. (2009). IETU / Obligación de enviar el listado de conceptos que sirvieron de base para determinarlo. México: IMCP. Disponible en http://imcp.org.mx/servicios/folios/folios-20082009/282008-2009-ietu-obligacion-de-enviar-el-listado-de-conceptos-que-sirvieron-de-base-para-determinarlo/ Consultado: 09/08/2017

Instituto Mexicano del Seguro Social. (1992). Diario Oficial de la Federación 28/10/1992. Instructivo para la dictaminación sobre el cumplimiento de las obligaciones que la ley del seguro social y sus reglamentos imponen a los patrones y demás sujetos obligados. México: Secretaría de Gobernación. Disponible en http://dof.gob.mx/ nota_detalle.php? $\operatorname{codigo}=4695587 \&$ fecha $=28 / 10 / 1992$ Consultado: $21 / 08 / 2017$ 


\section{S. Lagunas Puls y E. L. León Vite / Contaduría y Administración 65(2) 2020, 1-18 \\ http://dx.doi.org/10.22201/fca.24488410e.2018.1867}

Israel, R., Rosenthal, J., \& Wei, J. (2001). Finding generators for Markov Chains via empirical transition matrices, with applications to credit ratings. Mathematical Finance Vol. 11 No. 2, 245-265. Disponible en ftp://137.82.36.73/pub/israel/irw.pdf Consultado: 20/09/2017

León, E., \& Lagunas, S. (2017). Auditoría forense: Conceptualizaciones y adopción en América Latina. RECAI Revista de Estudios en Contaduría, Administración e Informática, 1-23. Disponible en https://recai.uaemex.mx/ article/view/8918 Consultado: 22/10/2017

Levin, D., Peres, Y., \& Wilmert, E. (2007). Markov Chains and Mixing Times. USA: Mathematical Sciences Research Institute, the National Science Foundation VIGRE grant to the Department of Statistics at the University of Berkeley. Disponible en https://s3.amazonaws.com/academia.edu.documents/30694248/recent.pdf?AWSAccessKeyId=AKIAIWOWYYGZ2Y53UL3A\&Expires $=1507244079 \&$ Signature $=$ Bb $\% 2$ FPO7QXfK $\%$ 2F1MfcXBqTv9pMIPDE\%3D\&response-content-disposition=inline\%3B\%20filename\%3DMarkov_chains_ and_mixing_times.pdf Consultado: 16/09/2017

Norris, J. (1998). Markov chains. UK: Cambridge university press.

Ordoñez, Y. J., Lerma, L. F., \& Ocampo, E. M. (2008). Ordoñez, Y. J., Lerma, L. F., \& Ocampo, E. M. T. (2008). Aplicación de cadenas de markov continuas a las estadísticas del secuestro en colombia. Scientia et technica, 14(38), 235-240. Disponible en http://www.redalyc.org/pdf/849/84903841.pdf Consultado: 30/11/2017

Quah, D. (1996). Empirics for economic growth and convergence. European Economic Review 40 (6), 1353-1375. doi:https://doi.org/10.1016/0014-2921(95)00051-8 Consultado: 09/11/2017

Render, B., Stair, R., \& Hanna, M. (2012). Análisis de Markov. En B. Render, R. Stair, \& M. Hanna, Métodos cuantitativos para los negocios (págs. 574-586). México: Pearson Educación.

Secretaría de Hacienda y Crédito Público. (1998). Diario Oficial de la Federación 13/02/1998. Anexo 1 de la Resolución Miscelánea Fiscal para 1997. México: Secretaría de Gobernación. Disponible en http://www.dof.gob. $\mathrm{mx} /$ nota_detalle.php?codigo $=4865711 \&$ fecha $=13 / 02 / 1998$ Consultado: $02 / 08 / 2017$

Servicio de Administración Tributaria. (2016). Fiscalización electrónica. México: Secretaría de Hacienda y Crédito Público. Disponible en http://imcp.org.mx/wp-content/uploads/2016/09/Fiscalizaci\%C3\%B3n-Electr\%C3\%B3nica.pdf Consultado: 21/08/2017

Servicio de Administración Tributaria. (31 de 08 de 2017). Datos Abiertos. Disponible en Auditoría - Actos de Fiscalización - Por método: http://www.sat.gob.mx/cifras_sat/Paginas/datos/vinculo.html?page=ActPorMet.html

University of St Andrews. (05 de 10 de 2017). School of Mathematics and Statistics. Disponible en Andrei Andreyevich Markov: http://www-history.mcs.st-andrews.ac.uk/Biographies/Markov.html 